\title{
Prize-Collecting Steiner Network Problems
}

\author{
MohammadTaghi Hajiaghayi $^{1}$, Rohit Khandekar ${ }^{2}$, Guy Kortsarz $^{3}{ }^{\star}$, and Zeev Nutov ${ }^{4}$ \\ 1 ATT Research Lab Research. Hajiagha@research.att.com \\ 2 IBM T.J.Watson Research Center. rohitk@us.ibm.com \\ 3 Rutgers University, Camden. guyk@camden.rutgers.edu \\ 4 The Open University of Israel nutov@openu.ac.il
}

\begin{abstract}
In the Steiner Network problem we are given a graph $G$ with edge-costs and connectivity requirements $r_{u v}$ between node pairs $u, v$. The goal is to find a minimum-cost subgraph $H$ of $G$ that contains $r_{u v}$ edge-disjoint paths for all $u, v \in V$. In Prize-Collecting Steiner Network problems we do not need to satisfy all requirements, but are given a penalty function for violating the connectivity requirements, and the goal is to find a subgraph $H$ that minimizes the cost plus the penalty. The case when $r_{u v} \in\{0,1\}$ is the classic Prize-Collecting Steiner Forest problem.

In this paper we present a novel linear programming relaxation for the Prize-Collecting Steiner Network problem, and by rounding it, obtain the first constant-factor approximation algorithm for submodular and monotone non-decreasing penalty functions. In particular, our setting includes all-or-nothing penalty functions, which charge the penalty even if the connectivity requirement is slightly violated; this resolves an open question posed in [SSW07]. We further generalize our results for element-connectivity and node-connectivity.
\end{abstract}

\footnotetext{
* Partially supported by NSF Award Grant number 0829959.
} 


\section{Introduction}

Prize-collecting Steiner problems are well-known network design problems with several applications in expanding telecommunications networks (see e.g. [JMP00,SCRS00]), cost sharing, and Lagrangian relaxation techniques (see e.g. [JV01,CRW01]). A general form of these problems is the Prize-Collecting Steiner Forest problem $^{1}$ : given a network (graph) $G=(V, E)$, a set of source-sink pairs $\mathcal{P}=\left\{\left\{s_{1}, t_{1}\right\},\left\{s_{2}, t_{2}\right\}, \ldots,\left\{s_{k}, t_{k}\right\}\right\}$, a non-negative cost function $c: E \rightarrow \Re_{+}$, and a non-negative penalty function $\pi: \mathcal{P} \rightarrow \Re_{+}$, our goal is a minimum-cost way of installing (buying) a set of links (edges) and paying the penalty for those pairs which are not connected via installed links. When all penalties are $\infty$, the problem is the classic APX-hard Steiner Forest problem, for which the best known approximation ratio is $2-\frac{2}{n}$ ( $n$ is the number of nodes of the graph) due to Agrawal, Klein, and Ravi [AKR95] (see also [GW95] for a more general result and a simpler analysis). The case of Prize-Collecting Steiner Forest problem when all sinks are identical is the classic Prize-Collecting Steiner Tree problem. Bienstock, Goemans, Simchi-Levi, and Williamson [BGSLW93] first considered this problem (based on a problem earlier proposed by Balas [Bal89]) and gave for it a 3approximation algorithm. The current best ratio for this problem is 1.992 by Archer, Bateni, Hajiaghayi, and Karloff [ABHK09], improving upon a primal-dual $\left(2-\frac{1}{n-1}\right)$-approximation algorithm of Goemans and Williamson [GW95]. When in addition all penalties are $\infty$, the problem is the classic Steiner Tree problem, which is known to be APX-hard [BP89] and for which the best approximation ratio is 1.55 [RZ05].

The general form of the Prize-Collecting Steiner Forest problem first has been formulated by Hajiaghayi and Jain [HJ06]. They showed how by a primal-dual algorithm to a novel integer programming formulation of the problem with doubly-exponential variables, we can obtain a 3-approximation algorithm for the problem. In addition, they show that the factor 3 in the analysis of their algorithm is tight. However they show how a direct randomized LP-rounding algorithm with approximation factor 2.54 can be obtained for this problem. Their approach has been generalized by Sharma, Swamy, and Williamson [SSW07] for network design problems where violated arbitrary 0-1 connectivity constraints are allowed in exchange for a very general penalty function. The work of Hajiaghayi and Jain has also motivated a game-theoretic version of the problem considered by Gupta et al. $\left[\mathrm{GKL}^{+} 07\right]$.

In this paper, we consider a much more general high-connectivity version of Prize-Collecting Steiner Forest, called Prize-Collecting Generalized Steiner Network, in which we are also given connectivity requirements $r_{u v}$ for pairs of nodes $u$ and $v$ and a penalty function in case we do not satisfy all $r_{u v}$. Our goal is to find a minimum way of constructing a network (graph) in which we connect $u$ and $v$ with $r_{u v}^{\prime} \leq r_{u v}$ edge-disjoint paths and paying a penalty for all violated connectivity between source-sink pairs. This problem can arise in real-world network design, in which a typical client not only might want to connect to the network but also might want to connected via a few disjoint paths (e.g., to have a higher bandwidth or redundant connections in case of edge failures) and a penalty might be charged if we cannot satisfy its connectivity requirement. When all penalties are $\infty$, the problem is the classic Steiner Network problem. Improving on a long line of earlier research that applied primal-dual methods, Jain [Jai01] obtained a 2-approximation algorithm for Steiner Network using the iterative rounding method. This algorithm was generalized to so called "element-connectivity" by Fleischer, Jain, and Williamson [FJW01] and by Cheriyan, Vempala, and Vetta [CVV06]. Recently, some results were obtained for the node-connectivity version; the currently best known ratios for the node-connectivity case are $O\left(R^{3} \log n\right)$ for general requirements [CK09] and $O\left(R^{2}\right)$ for rooted requirements [Nut09], where $R=\max _{u, v \in V} r_{u v}$ is the maximum requirement. See the survey by Kortsarz and Nutov [KN07] for various min-cost connectivity problems.

Hajiaghayi [Haj08] generalizes the iterative rounding approach of Jain to Prize-Collecting Steiner Network when there is a separate non-increasing marginal penalty function for each pair $u, v$ which $r_{u v}$-connectivity requirement is not satisfied. He obtains an iterative rounding 3 -approximation algorithm for this case. For the special case when penalty functions are linear in the violation of the connectivity requirements, Nagarajan, Sharma, and Williamson [NSW08] using Jains iterative rounding algorithm as a black box give a 2.54-factor

\footnotetext{
${ }^{1}$ In the literature, this problem is also called "prize-collecting generalized Steiner tree".
} 
approximation algorithm. They also generalize the 0-1 requirements of Prize-Collecting Steiner Forest problem introduced by Sharma, Swamy, and Williamson [SSW07] to include general connectivity requirements. Assuming the monotone submodular penalty function of Sharma et al. is generalized to a multiset function that can be decomposed into functions in the same type as that of Sharma et al., they give an $O(\log R)$ approximation algorithm (recall that $R$ is the maximum connectivity requirement). In this algorithm, they assume that we can use each edge possibly many times (without bound). They raise the question whether we can obtain a constant ratio without all these assumptions, when penalty is a submodular multi-set function of the set of disconnected pairs? More importantly they pose as an open problem to design a good approximation algorithm for the all-or-nothing version of penalty functions: penalty functions which charge the penalty even if the connectivity requirement is slightly violated. In this paper, we answer affirmatively all these open problems by proving the first constant factor 2.54-approximation algorithm which is based on a novel LP formulation of the problem. We further generalize our results for element-connectivity and nodeconnectivity. In fact, for all types of connectivities, we prove a very general result (see Theorem 1) stating that if Steiner Network (the version without penalties) admits an LP-based $\rho$-approximation algorithm, then the corresponding prize-collecting version admits a $(\rho+1)$-approximation algorithm.

\subsection{Problems we consider}

In this section, we define formally the terms used in the paper. For a subset $S$ of nodes in a graph $H$, let $\lambda_{H}^{S}(u, v)$ denote the $S$-connectivity between $u$ and $v$ in $H$, namely, the maximum number of edge-disjoint $u v$-paths in $H$ so that no two of them have a node in $S-\{u, v\}$ in common. In the Generalized Steiner-Network (GSN) problem we are given a graph $G=(V, E)$ with edge-costs $\left\{c_{e} \geq 0 \mid e \in E\right\}$, a node subset $S \subseteq V$, a collection $\left\{u_{1}, v_{1}\right\}, \ldots,\left\{u_{k}, v_{k}\right\}$ of node pairs from $V$, and $S$-connectivity requirements $r_{1}, \ldots, r_{k}$. The goal is to find a minimum cost subgraph $H$ of $G$ so that $\lambda_{H}^{S}\left(u_{i}, v_{i}\right) \geq r_{i}$ for all $i$. Extensively studied particular cases of GSN are: the Steiner Network problem, called also Edge-Connectivity GSN $(S=\emptyset)$, Node-Connectivity GSN $(S=V)$, and Element-Connectivity GSN $\left(S \cap\left\{u_{i}, v_{i}\right\}=\emptyset\right.$ for all $\left.i\right)$. The case of rooted requirements is when there is a "root" $s$ that belongs to all pairs $\left\{u_{i}, v_{i}\right\}$. We consider the following "prize-collecting" version of GSN.

\section{All-or-Nothing Prize Collecting Generalized Steiner Network (PC-GSN):}

Instance: A graph $G=(V, E)$ with edge-costs $\left\{c_{e} \geq 0 \mid e \in E\right\}, S \subseteq V$, a collection $\left\{u_{1}, v_{1}\right\}, \ldots,\left\{u_{k}, v_{k}\right\}$ of node pairs from $V, S$-connectivity requirements $r_{1}, \ldots, r_{k}>0$, and a penalty function $\pi: 2^{\{1, \ldots, k\}} \rightarrow \Re_{+}$.

Objective: Find a subgraph $H$ of $G$ that minimizes the value

$$
\operatorname{val}(H)=c(H)+\pi(\operatorname{unsat}(H))
$$

of $H$, where unsat $(H)=\left\{i \mid \lambda_{H}^{S}\left(u_{i}, v_{i}\right)<r_{i}\right\}$ is the set of requirements not satisfied by $H$.

We will assume that the penalty function $\pi$ is given by an evaluation oracle. We will also assume that $\pi$ is submodular, namely, that $\pi(A)+\pi(B) \geq \pi(A \cap B)+\pi(A \cup B)$ for all $A, B$ and that it is monotone non-decreasing, namely, $\pi(A) \leq \pi(B)$ for all $A, B$ with $A \subseteq B$. As was mentioned, approximating the edge-connectivity variant of PC-GSN was posed as the main open problem by Nagarajan, Sharma, and Williamson [NSW08]. We resolve this open problem for the submodular function val $(H)$ considered here.

We next define the second problem we consider. 
Generalized Steiner Network with Generalized Penalties (GSN-GP):

Instance: A graph $G=(V, E)$ with edge-costs $\left\{c_{e} \geq 0 \mid e \in E\right\}, S \subseteq V$, a collection $\left\{u_{1}, v_{1}\right\}, \ldots,\left\{u_{k}, v_{k}\right\}$ of node pairs from $V$, and non-increasing penalty functions $p_{1}, \ldots, p_{k}:\{0,1, \ldots, n-1\} \rightarrow \Re_{+}$.

Objective: Find a subgraph $H$ of $G$ that minimizes the value

$$
\operatorname{val}^{\prime}(H)=c(H)+\sum_{i=1}^{k} p_{i}\left(\lambda_{H}^{S}\left(u_{i}, v_{i}\right)\right) .
$$

The above problem captures general penalty functions of the $S$-connectivity $\lambda^{S}\left(u_{i}, v_{i}\right)$ for given pairs $\left\{u_{i}, v_{i}\right\}$. It is natural to assume that the penalty functions are non-increasing, i.e., we pay less in the objective function if the achieved connectivity is more. This problem was posed as an open question by Nagarajan et al. [NSW08]. In this paper, we use the convention that $p_{i}(n)=0$ for all $i$.

We need some definitions to introduce our results. A pair $T=\left\{T^{\prime}, T^{\prime \prime}\right\}$ of subsets of $V$ is called a setpair (of $V$ ) if $T^{\prime} \cap T^{\prime \prime}=\emptyset$. Let $K=\{1, \ldots k\}$. Let $T=\left\{T^{\prime}, T^{\prime \prime}\right\}$ be a setpair of $V$. We denote by $\delta(T)$ the set of edges in $E$ between $T^{\prime}$ and $T^{\prime \prime}$. For $i \in K$ we use $T \odot(i, S)$ to denote that $\left|T^{\prime} \cap\left\{u_{i}, v_{i}\right\}\right|=1,\left|T^{\prime \prime} \cap\left\{u_{i}, v_{i}\right\}\right|=1$ and $V \backslash\left(T^{\prime} \cup T^{\prime \prime}\right) \subseteq S$. While in the case of edge-connectivity a "cut" consists of edges only, in the case of $S$-connectivity a cut that separates between $u$ and $v$ is "mixed", meaning it may contain both edges in the graph and nodes from $S$. Note that if $T \odot(i, S)$ then $\delta(T) \cup\left(V \backslash\left(T^{\prime} \cup T^{\prime \prime}\right)\right)$ is such a mixed cut that separates between $u_{i}$ and $v_{i}$. Intuitively, Menger's Theorem for $S$-connectivity (c.f. [KN07]) states that the $S$-connectivity between $u_{i}$ and $v_{i}$ equals the minimum cardinality of such a mixed cut. Formally, for a node pair $u_{i}, v_{i}$ of a graph $H=(V, E)$ and $S \subseteq V$ we have:

$$
\lambda_{H}^{S}\left(u_{i}, v_{i}\right)=\min _{T \odot(i, S)}\left(|\delta(T)|+\left|V \backslash\left(T^{\prime} \cup T^{\prime \prime}\right)\right|\right)=\min _{T \odot(i, S)}\left(|\delta(T)|+|V|-\left(\left|T^{\prime}\right|+\left|T^{\prime \prime}\right|\right)\right)
$$

Hence if $\lambda_{H}^{S}\left(u_{i}, v_{i}\right) \geq r_{i}$ for a graph $H=(V, E)$, then for any setpair $T$ with $T \odot(i, S)$ we must have $|\delta(T)| \geq r_{i}(T)$, where $r_{i}(T)=\max \left\{r_{i}+\left|T^{\prime}\right|+\left|T^{\prime \prime}\right|-|V|, 0\right\}$. Consequently, a standard "cut-type" LPrelaxation of the GSN problem is as follows (c.f. [KN07]).

$$
\min \left\{\sum_{e \in E} c_{e} x_{e} \mid \sum_{e \in \delta(T)} x_{e} \geq r_{i}(T) \forall T \odot(i, S), \forall i \in K, x_{e} \in[0,1] \forall e\right\} .
$$

\subsection{Our results}

We introduce a novel LP relaxation of the problem which is shown to be better, in terms of the integrality gap, than a "natural" LP relaxation considered in [NSW08]. Using our LP relaxation, we prove the following main result.

Theorem 1. Suppose that there exists a polynomial time algorithm that computes an integral solution to LP (1) of cost at most $\rho$ times the optimal value of LP (1) for any subset of node pairs. Then PC-GSN admits $a\left(1-e^{-1 / \rho}\right)^{-1}$-approximation algorithm, provided that the penalty function $\pi$ is submodular and monotone non-decreasing.

Note that since $1-\frac{1}{\rho}<e^{-\frac{1}{\rho}}<1-\frac{1}{\rho+1}$ holds for $\rho \geq 1$, we have $\rho<\left(1-e^{-1 / \rho}\right)^{-1}<\rho+1$.

Let $R=\max _{i} r_{i}$ denote the maximum requirement. The best known values of $\rho$ are as follows: 2 for Edge-GSN [Jai01], 2 for Element-GSN [FJW01,CVV06], $O\left(R^{3} \log |V|\right)$ for Node-GSN [CK09], and $O\left(R^{2}\right)$ for Node-GSN with rooted requirements [Nut09]. Substituting these values in Theorem 1, we obtain: 
Corollary 1. PC-GSN problems admit the following approximation ratios provided that the penalty function $\pi$ is submodular and monotone non-decreasing: 2.54 for edge-connectivity and element-connectivity, $O\left(R^{3} \log |V|\right)$ for node-connectivity, and $O\left(R^{2}\right)$ for node-connectivity with rooted requirements.

Our results for GSN-GP follow from Corollary 1.

Corollary 2. GSN-GP problems admit the following approximation ratios: 2.54 for edge-connectivity and element-connectivity, $O\left(R^{3} \log |V|\right)$ for node-connectivity, and $O\left(R^{2}\right)$ for node-connectivity with rooted requirements. Here $R=\max _{1 \leq i \leq k} \min \left\{\lambda \geq 0 \mid p_{i}(\lambda)=0\right\}$.

Proof. We present an approximation ratio preserving reduction from the GSN-GP problem to the corresponding PC-GSN problem. Given an instance of the GSN-GP problem, we create an instance of the PC-GSN problem as follows. The PC-GSN instance inherits the graph $G$, its edge-costs, and the set $S$. Let $\left(u_{i}, v_{i}\right)$ be a pair in GSN-GP and let $R_{i}=\min \left\{\lambda \geq 0 \mid p_{i}(\lambda)=0\right\}$. We introduce $R_{i}$ copies of this pair, $\left\{\left(u_{i}^{1}, v_{i}^{1}\right), \ldots,\left(u_{i}^{R_{i}}, v_{i}^{R_{i}}\right)\right\}$, to the set of pairs in the PC-GSN instance. We set the edge-connectivity requirement of a pair $\left(u_{i}^{t}, v_{i}^{t}\right)$ to be $t$ for $1 \leq t \leq R_{i}$. We also set the penalty function for singleton sets as follows $\pi\left(\left\{\left(u_{i}^{t}, v_{i}^{t}\right)\right\}\right)=p_{i}(t-1)-p_{i}(t)$ for all $1 \leq t \leq R_{i}$. Finally, we extend this function $\pi$ to a set of pairs $P$ by linearity, i.e., $\pi(P)=\sum_{p \in P} \pi(\{p\})$. Note that such a function $\pi$ is clearly submodular and monotone non-decreasing.

It is sufficient to show that for any subgraph $H$ of $G$, its value in the GSN-GP instance equals its value in the PC-GSN instance, i.e., $\operatorname{val}(H)=\operatorname{val}^{\prime}(H)$; then we can use the algorithm from Corollary 1 to complete the proof. Fix a pair $\left(u_{i}, v_{i}\right)$. Let $\lambda_{H}^{S}\left(u_{i}, v_{i}\right)=t_{i}$. Thus the contribution of pair $\left(u_{i}, v_{i}\right)$ to the objective function $\operatorname{val}^{\prime}(H)$ of the GSN-GP instance is $p_{i}\left(t_{i}\right)$. On the other hand, since $\pi$ is linear, the total contribution of pairs $\left\{\left(u_{i}^{1}, v_{i}^{1}\right), \ldots,\left(u_{i}^{R_{i}}, v_{i}^{R_{i}}\right)\right\}$ to the objective function val' $(H)$ of the PC-GSN instance is $\sum_{t=t_{i}+1}^{R_{i}} \pi\left(\left\{\left(u_{i}^{t}, v_{i}^{t}\right)\right\}\right)=\sum_{t=t_{i}+1}^{R_{i}}\left(p_{i}(t-1)-p_{i}(t)\right)=p_{i}\left(t_{i}\right)$. Summing up over all pairs, we conclude that $\operatorname{val}(H)=\operatorname{val}^{\prime}(H)$, as claimed.

\section{A new $\mathrm{LP}$ relaxation}

We use the following LP-relaxation for the PC-GSN problem. We introduce variables $x_{e}$ for $e \in E\left(x_{e}=1\right.$ if $e \in H), f_{i, e}$ for $i \in K$ and $e \in E\left(f_{i, e}=1\right.$ if $i \notin$ unsat $(H)$ and $e$ appears on a chosen set of $r_{i} S$-disjoint $\left\{u_{i}, v_{i}\right\}$-paths in $\left.H\right)$, and $z_{I}$ for $I \subseteq K\left(z_{I}=1\right.$ if $I=$ unsat $\left.(H)\right)$.

$$
\begin{aligned}
\text { Minimize } & \sum_{e \in E} c_{e} x_{e}+\sum_{I \subseteq K} \pi(I) z_{I} & & \\
\text { Subject to } & \sum_{e \in \delta(T)} f_{i, e} \geq\left(1-\sum_{I: i \in I} z_{I}\right) r_{i}(T) & & \forall i \quad \forall T \odot(i, S) \\
f_{i, e} & \leq 1-\sum_{I: i \in I} z_{I} & & \forall i \quad \forall e \\
x_{e} & \geq f_{i, e} & & \forall i \quad \forall e \\
\sum_{I \subseteq K} z_{I} & =1 & & \\
x_{e}, f_{i, e}, z_{I} & \in[0,1] & & \forall i \quad \forall e \quad \forall I
\end{aligned}
$$

We first prove that (2) is a valid LP-relaxation of the PC-GSN problem.

Lemma 1. The optimal value of LP (2) is at most the optimal solution value to the PC-GSN problem. Moreover, if $\pi$ is monotone non-decreasing, the optimum solution value to the PC-GSN problem is at most the value of the optimum integral solution of LP (2). 
Proof. Given a feasible solution $H$ to the PC-GSN problem define a feasible solution to LP (2) as follows. Let $x_{e}=1$ if $e \in H$ and $x_{e}=0$ otherwise. Let $z_{I}=1$ if $I=$ unsat $(H)$ and $z_{I}=0$ otherwise. For each $i \in \operatorname{unsat}(H)$ set $f_{i, e}=0$ for all $e \in E$, while for $i \notin$ unsat $(H)$ the variables $f_{i, e}$ take values as follows: fix a set of $r_{i}$ pairwise $S$-disjoint $\left\{u_{i}, v_{i}\right\}$-paths, and let $f_{i, e}=1$ if $e$ belongs to one of these paths and $f_{i, e}=0$ otherwise. The defined solution is feasible for LP (2): the first set of constraints are satisfied by Menger's Theorem for $S$-connectivity, while the remaining constraints are satisfied by the above definition of variables. It is also easy to see that the above solution has value exactly val $(H)$.

If $\pi$ is monotone non-decreasing, we prove that for any integral solution $\left\{x_{e}, f_{i, e}, z_{I}\right\}$ to (2), the graph $H$ with edge-set $\left\{e \in E \mid x_{e}=1\right\}$ has $\operatorname{val}(H)$ at most the value of the solution $\left\{x_{e}, f_{i, e}, z_{I}\right\}$. To see this, first note that there is a unique set $I \subseteq K$ with $z_{I}=1$, since the variables $z_{I}$ are integral and $\sum_{I \subset K} z_{I}=1$. Now consider an index $i \notin I$. Since $\sum_{I: i \in I} z_{I}=0$, we have $\sum_{e \in \delta(T)} x_{e} \geq \sum_{e \in \delta(T)} f_{i, e} \geq r_{i}(T)$ for all $T \odot(i, S)$. This implies that $i \notin$ unsat $(H)$, by Menger's Theorem for $S$-connectivity. Consequently, unsat $(H) \subseteq I$, hence $\pi($ unsat $(H)) \leq \pi(I)$ by the monotonicity of $\pi$. Thus $\operatorname{val}(H)=c(H)+\pi($ unsat $(H)) \leq$ $\sum_{e \in E} c_{e} x_{e}+\sum_{I \subseteq K} \pi(I) z_{I}$ and the lemma follows.

\subsection{Why a "natural" LP relaxation does not work?}

One may be tempted to consider a natural LP without using the flow variables $f_{i, e}$, namely, the LP obtained from LP (2) by replacing the the first three sets of constraints by the set of constraints

$$
\sum_{e \in \delta(T)} x_{e} \geq\left(1-\sum_{I: i \in I} z_{I}\right) r_{i}(T)
$$

for all $i$ and $T \odot(i, S)$. Here is an example demonstrating that the integrality gap of this LP can be as large as $R=\max _{i} r_{i}$ even for edge-connectivity. Let $G$ consist of $R-1$ edge-disjoint paths between two nodes $s$ and $t$. All the edges have cost 0 . There is only one pair $\left\{u_{1}, v_{1}\right\}=\{s, t\}$ that has requirement $r_{1}=R$ and penalty $\pi(\{1\})=1$. Let $\pi(\emptyset)=0$. Clearly, $\pi$ is submodular and monotone non-decreasing. We have $S=\emptyset$. No integral solution can satisfy the requirement $r_{1}$, hence an optimal integral solution pays the penalty $\pi(\{1\})$ and has value 1. A feasible fractional solution (without the flow variables) sets $x_{e}=1$ for all $e$, and sets $z_{\{1\}}=1 / R$, $z_{\emptyset}=1-1 / R$. The new set of constraints is satisfied since $\sum_{e \in \delta(T)} x_{e} \geq(1-1 / R) \cdot R=\left(1-z_{\{1\}}\right) r_{1}(T)$ for any $\{s, t\}$-cut $T$. Thus the optimal LP-value is at most $1 / R$, giving a gap of at least $R$.

With flow variables, however, we have an upper bound $f_{1, e} \leq 1-z_{\{1\}}$. Since there is an $\{s, t\}$-cut $T$ with $|\delta(T)|=R-1$, we cannot satisfy the constraints $\sum_{e \in \delta(T)} f_{1, e} \geq\left(1-z_{\{1\}}\right) r_{1}(T)$ and $f_{1, e} \leq 1-z_{\{1\}}$ simultaneously unless we set $z_{\{1\}}=1$. Thus in this case, our LP (2) with flow variables has the same optimal value of as the integral optimum.

\subsection{Some technical results regarding LP (2)}

We will prove the following two statements that together imply Theorem 1.

Lemma 2. Any basic feasible solution to (2) has a polynomial number of non-zero variables. Furthermore, an optimal basic solution to (2) (the non-zero entries) can be computed in polynomial time.

Lemma 3. There exists a polynomial time algorithm that given a feasible solution to (2) computes a subgraph $H$ of $G$ so that $\operatorname{val}(H)=c(H)+\pi(\operatorname{unsat}(H))$ is at most $\left(1-e^{-1 / \rho}\right)^{-1}$ times the value of this solution, assuming $\pi$ is submodular and monotone non-decreasing. 
Before proving these lemmas, we prove some useful results.

Lemma 4. Let $\gamma \in[0,1]^{k}$ be a vector. Consider a primal $L P$

$$
\mathrm{P}(\gamma):=\min \left\{\sum_{I \subseteq K} \pi(I) z_{I} \mid \sum_{I: i \in I} z_{I} \geq \gamma_{i} \forall i \in K, z_{I} \geq 0 \forall I \subseteq K\right\}
$$

and its dual LP

$$
\mathrm{D}(\gamma):=\max \left\{\sum_{i \in K} \gamma_{i} y_{i} \mid \sum_{i \in I} y_{i} \leq \pi(I) \forall I \subseteq K, y_{i} \geq 0 \forall i \in K\right\} .
$$

Let $\rho$ be a permutation of $K$ such that $\gamma_{\rho(1)} \leq \gamma_{\rho(2)} \leq \ldots \leq \gamma_{\rho(k)}$. Let us also use the notation that $\gamma_{\rho(0)}=0$. The optimum solutions to $\mathrm{P}(\gamma)$ and $\mathrm{D}(\gamma)$ respectively are given by

$$
z_{I}= \begin{cases}\gamma_{\rho(i)}-\gamma_{\rho(i-1)}, & \text { for } I=\{\rho(i), \ldots, \rho(k)\}, i \in K \\ 0, & \text { otherwise }\end{cases}
$$

and

$$
y_{\rho(i)}=\pi(\{\rho(i), \ldots, \rho(k)\})-\pi(\{\rho(i+1), \ldots, \rho(k)\}), \text { for } i \in K .
$$

Proof. To simplify the notation, we assume without loss of generality that $\gamma_{1} \leq \gamma_{2} \leq \cdots \leq \gamma_{k}$, i.e., that $\rho$ is the identity permutation.

We argue that the above defined $\left\{z_{I}\right\}$ and $\left\{y_{i}\right\}$ form feasible solutions to the primal and dual LPs respectively. Note that $z_{I} \geq 0$ for all $I$ and $\sum_{I: i \in I} z_{I}=\sum_{j=1}^{i}\left(\gamma_{j}-\gamma_{j-1}\right)=\gamma_{i}$ for all $i$. Since $\pi$ is monotone non-decreasing, the above defined $y_{i}$ satisfy $y_{i} \geq 0$ for all $i$. Now fix $I \subseteq K$. Let $I=\left\{i_{1}, \ldots, i_{p}\right\}$ where $i_{1}<\cdots<i_{p}$. Therefore

$$
\begin{aligned}
\sum_{i \in I} y_{i}=\sum_{j=1}^{p} y_{i_{j}} & =\sum_{j=1}^{p}\left[\pi\left(\left\{i_{j}, \ldots, k\right\}\right)-\pi\left(\left\{i_{j}+1, \ldots, k\right\}\right)\right] \\
& \leq \sum_{j=1}^{p}\left[\pi\left(\left\{i_{j}, i_{j+1}, \ldots, i_{p}\right\}\right)-\pi\left(\left\{i_{j+1}, i_{j+2}, \ldots, i_{p}\right\}\right)\right] \\
& =\pi\left(\left\{i_{1}, \ldots, i_{p}\right\}\right)=\pi(I) .
\end{aligned}
$$

The above inequality holds because of the submodularity of $\pi$. Next observe that the solutions $\left\{z_{I}\right\}$ and $\left\{y_{i}\right\}$ satisfy

$$
\begin{aligned}
\sum_{I} \pi(I) z_{I} & =\sum_{i=1}^{k} \pi(\{i, \ldots, k\}) \cdot\left(\gamma_{i}-\gamma_{i-1}\right) \\
& =\sum_{i=1}^{k} \gamma_{i} \cdot(\pi(\{i, \ldots, k\})-\pi(\{i+1, \ldots, k\})) \\
& =\sum_{i} \gamma_{i} y_{i} .
\end{aligned}
$$

Thus from weak LP duality, they in fact form optimum solutions to primal and dual LPs respectively.

Recall that a sub-gradient of a convex function $g: \Re^{k} \rightarrow \Re$ at a point $\gamma \in \Re^{k}$ is a vector $d \in \Re^{k}$ such that for any $\gamma^{\prime} \in \Re^{k}$, we have $g\left(\gamma^{\prime}\right)-g(\gamma) \geq d \cdot\left(\gamma^{\prime}-\gamma\right)$. For a differentiable convex function $g$, the sub-gradient corresponds to gradient $\nabla g$. 
Lemma 5. The function $\mathrm{P}:[0,1]^{k} \rightarrow \Re_{+}$, defined in Lemma 4, is convex and given $\gamma \in[0,1]^{k}$, both $\mathrm{P}(\gamma)$ and its sub-gradient $\nabla \mathrm{P}(\gamma)$ can be computed efficiently in polynomial time.

Proof. We first prove that P is convex. Fix $\gamma_{1}, \gamma_{2} \in[0,1]^{k}$ and $\alpha \in[0,1]$. To show that $\mathrm{P}$ is convex, we will show $P\left(\alpha \gamma_{1}+(1-\alpha) \gamma_{2}\right) \leq \alpha \mathrm{P}\left(\gamma_{1}\right)+(1-\alpha) \mathrm{P}\left(\gamma_{2}\right)$. Let $\left\{z_{I}^{1}\right\}$ and $\left\{z_{I}^{2}\right\}$ be the optimum solutions of the primal LP defining $\mathrm{P}$ for $\gamma_{1}$ and $\gamma_{2}$ respectively. Note that the solution $\left\{\alpha z_{I}^{1}+(1-\alpha) z_{I}^{2}\right\}$ is feasible for this LP for $\gamma=\alpha \gamma_{1}+(1-\alpha) \gamma_{2}$. Thus the optimum solution has value not greater than the value of this solution which is $\alpha \mathrm{P}\left(\gamma_{1}\right)+(1-\alpha) \mathrm{P}\left(\gamma_{2}\right)$.

From Lemma 4, it is clear that given $\gamma \in[0,1]^{k}$, the value $\mathrm{P}(\gamma)$ can be computed in polynomial time. Lemma 4 also implies that the optimum dual solution $y^{*}=\left(y_{1}^{*}, \ldots, y_{k}^{*}\right) \in \Re_{+}^{k}$ can be computed in polynomial time. We now argue that $y^{*}$ is a sub-gradient of P at $\gamma$. Fix any $\gamma^{\prime} \in \Re^{k}$. First note that, from LP duality, $\mathrm{P}(\gamma)=y^{*} \cdot \gamma$. Thus we have

$$
\mathrm{P}(\gamma)+y^{*} \cdot\left(\gamma^{\prime}-\gamma\right)=y^{*} \cdot \gamma+y^{*} \cdot\left(\gamma^{\prime}-\gamma\right)=y^{*} \cdot \gamma^{\prime} \leq \mathrm{P}\left(\gamma^{\prime}\right)
$$

The last inequality holds since $y^{*}$ is a feasible solution for the LP $\mathrm{P}\left(\gamma^{\prime}\right)$ as well. The lemma follows.

\section{Proof of Lemma 3}

We now describe how to round LP $(2)$ solutions to obtain a $(\rho+1)$-approximation for PC-GSN. Later we show how to improve it to $\left(1-e^{-1 / \rho}\right)^{-1}$. Let $\left\{x_{e}^{*}, f_{i, e}^{*}, z_{I}^{*}\right\}$ be a feasible solution to LP $(2)$. Let $\alpha \in(0,1)$ be a parameter to be fixed later. We partition the requirements into two classes: we call a requirement $i \in K \operatorname{good}$ if $\sum_{I: i \in I} z_{I}^{*} \leq \alpha$ and bad otherwise. Let $K_{g}$ denote the set of good requirements. The following statement shows how to satisfy the good requirements.

Lemma 6. There exists a polynomial time algorithm that computes a subgraph $H$ of $G$ of cost $c(H) \leq$ $\frac{\rho}{1-\alpha} \cdot \sum_{e} c_{e} x_{e}^{*}$ that satisfies all good requirements.

Proof. Consider the LP-relaxation (1) of the GSN problem with good requirements only, with $K$ replaced by $K_{g}$; namely, we seek a minimum cost subgraph $H$ of $G$ that satisfies the set $K_{g}$ of good requirements. We claim that $x_{e}^{* *}=\min \left\{1, x_{e}^{*} /(1-\alpha)\right\}$ for each $e \in E$ is a feasible solution to LP (1). Thus the optimum value of LP (1) is at most $\sum_{e \in E} c_{e} x_{e}^{* *}$. Consequently, using the algorithm that computes an integral solution to LP (1) of cost at most $\rho$ times the optimal value of LP (1), we can construct a subgraph $H$ that satisfies all good requirements and has cost at most $c(H) \leq \rho \sum_{e \in E} c_{e} x_{e}^{* *} \leq \frac{\rho}{1-\alpha} \sum_{e} c_{e} x_{e}^{*}$, as desired.

We now show that $\left\{x_{e}^{* *}\right\}$ is a feasible solution to LP (1), namely, that $\sum_{e \in \delta(T)} x_{e}^{* *} \geq r_{i}(T)$ for any $i \in K_{g}$ and any $T \odot(i, S)$. Let $i \in K_{g}$ and let $\zeta_{i}=1-\sum_{I: i \in I} z_{I}^{*}$. Note that $\zeta_{i} \geq 1-\alpha$, by the definition of $K_{g}$. By the second and the third sets of constraints in $\operatorname{LP}(2)$, for every $e \in E$ we have $\min \left\{\zeta_{i}, x_{e}^{*}\right\} \geq f_{i, e}^{*}$. Thus we obtain:

$$
x_{e}^{* *}=\min \left\{1, \frac{x_{e}^{*}}{1-\alpha}\right\}=\frac{1}{\zeta_{i}} \min \left\{\zeta_{i}, \frac{\zeta_{i}}{1-\alpha} x_{e}^{*}\right\} \geq \frac{1}{\zeta_{i}} \min \left\{\zeta_{i}, x_{e}^{*}\right\} \geq \frac{f_{i, e}^{*}}{\zeta_{i}}=\frac{f_{i, e}^{*}}{1-\sum_{I: i \in I} z_{I}^{*}} .
$$

Consequently, combining with the first set of constraints in LP $(2)$ we obtain that for any $T \odot(i, S)$ :

$$
\sum_{e \in \delta(T)} x_{e}^{* *} \geq \frac{\sum_{e \in \delta(T)} f_{i, e}^{*}}{1-\sum_{I: i \in I} z_{I}^{*}} \geq r_{i}(T) .
$$


Let $H$ be as in Lemma 6 , and recall that unsat $(H)$ denotes the set of requirements not satisfied by $H$. Clearly each requirement $i \in$ unsat $(H)$ is bad. The following lemma bounds the total penalty we pay for unsat $(H)$.

Lemma 7. $\pi($ unsat $(H)) \leq \frac{1}{\alpha} \cdot \sum_{I} \pi(I) z_{I}^{*}$.

Proof. Define $\gamma \in[0,1]^{k}$ as follows: $\gamma_{i}=1$ if $i \in$ unsat $(H)$ and 0 otherwise. Now consider LP P $(\gamma)$ defined in Lemma 4. Since each $i \in \operatorname{unsat}(H)$ is bad, from the definition of bad requirements, it is clear that $\left\{z_{I}^{*} / \alpha\right\}$ is a feasible solution to LP $\mathrm{P}(\gamma)$. Furthermore, from Lemma 4, the solution $\left\{z_{I}\right\}$ defined as $z_{I}=1$ if $I=\operatorname{unsat}(H)$ and 0 otherwise is the optimum solution to $\mathrm{P}(\gamma)$. The cost of this solution, $\pi(\operatorname{unsat}(H))$, is therefore at most the cost of the feasible solution $\left\{z_{I}^{*} / \alpha\right\}$ which is $\frac{1}{\alpha} \cdot \sum_{I} \pi(I) z_{I}^{*}$. The lemma thus follows.

Combining Lemmas 6 and 7 , we obtain $\max \left\{\frac{\rho}{1-\alpha}, \frac{1}{\alpha}\right\}$-approximation. If we substitute $\alpha=1 /(\rho+1)$, we obtain a $(\rho+1)$-approximation for PC-GSN.

Improving the approximation to $\left(1-e^{-1 / \rho}\right)^{-1}$. We use a technique introduced by Goemans as follows. We pick $\alpha$ uniformly at random from the interval $(0, \beta]$ where $\beta=1-e^{-1 / \rho}$. From Lemmas 6 and 7 , the expected cost of the solution is at most

$$
\mathbb{E}_{\alpha}\left[\frac{\rho}{1-\alpha}\right] \cdot \sum_{e \in E} c_{e} x_{e}^{*}+\mathbb{E}_{\alpha}[\pi(\operatorname{unsat}(H))]
$$

To complete the proof of $\frac{1}{\beta}$-approximation, we now argue that the above expectation is at most $\frac{1}{\beta}$. $\sum_{e \in E}\left(c_{e} x_{e}^{*}+\sum_{I} \pi(I) z_{I}^{*}\right)$.

Since $\mathbb{E}_{\alpha}\left[\frac{\rho}{1-\alpha}\right]=\frac{1}{\beta}$, the first term in (3) is at most $\frac{1}{\beta} \cdot \sum_{e \in E} c_{e} x_{e}^{*}$. Since unsat $(H) \subseteq\left\{i \mid \sum_{I: i \in I} z_{I}^{*} \geq \alpha\right\}$ and since $\pi$ is monotone non-decreasing, the second term in (3) is at most $\mathbb{E}_{\alpha}\left[\pi\left(\left\{i \mid \sum_{I: i \in I} z_{I}^{*} \geq \alpha\right\}\right)\right]$. Lemma 8 bounds this quantity as follows. The ideas used here are also presented in Sharma et al. [SSW07].

Lemma 8. We have

$$
\mathbb{E}_{\alpha}\left[\pi\left(\left\{i \mid \sum_{I: i \in I} z_{I}^{*} \geq \alpha\right\}\right)\right] \leq \frac{1}{\beta} \cdot \sum_{I} \pi(I) z_{I}^{*} .
$$

Proof. Let $\gamma_{i}=\sum_{I: i \in I} z_{I}^{*}$ for all $i \in K$. Let us, without loss of generality, order the elements $i \in K$ such that $\gamma_{1} \leq \gamma_{2} \leq \cdots \leq \gamma_{k}$. We also use the notation $\gamma_{0}=0$. Note that $\left\{z_{I}^{*}\right\}$ forms a feasible solution to the primal LP $\mathrm{P}(\gamma)$ given in Lemma 4. Therefore, from Lemma 4, its objective value is at least that of the optimum solution:

$$
\sum_{I} \pi(I) z_{I}^{*} \geq \sum_{i=1}^{k}\left[\left(\gamma_{i}-\gamma_{i-1}\right) \cdot \pi(\{i, \ldots, k\})\right] .
$$

We now observe that the LHS of (4) can be expressed as follows. Since $\alpha$ is picked uniformly at random from $(0, \beta]$, we have that for all $1 \leq i \leq k$, with probability at most $\frac{\gamma_{i}-\gamma_{i-1}}{\beta}$, the random variable $\alpha$ lies in the interval $\left(\gamma_{i-1}, \gamma_{i}\right]$. When this event happens, we get that $\left\{i^{\prime} \mid \sum_{I: i^{\prime} \in I} z_{I}^{*} \geq \alpha\right\}=\left\{i^{\prime} \mid \gamma_{i^{\prime}} \geq \alpha\right\}=\{i, \ldots, k\}$. Thus the expectation in LHS of (4) is at most

$$
\sum_{i=1}^{k}\left[\frac{\gamma_{i}-\gamma_{i-1}}{\beta} \cdot \pi(\{i, \ldots, k\})\right] .
$$

From expressions (5) and (6), the lemma follows.

Thus the proof of $\left(1-e^{-1 / \rho}\right)^{-1}$-approximation is complete. 


\section{Proof of Lemma 2}

We next show that even if LP (2) has exponential number of variables and constraints, the following lemma holds.

Lemma 9. Any basic feasible solution to LP (2) has a polynomial number of non-zero variables.

Proof. Fix a basic feasible solution $\left\{x_{e}^{*}, f_{i, e}^{*}, z_{i}^{*}\right\}$ to (2). For $i \in K$, let

$$
\gamma_{i}=1-\frac{\min _{T: T \odot i} \sum_{e \in \delta(T)} f_{i, e}^{*}}{r_{i}} \quad \text { and } \quad \gamma_{i}^{\prime}=1-\max _{e} f_{i, e}^{*} .
$$

Now fix the values of variables $\left\{x_{e}, f_{i, e}\right\}$ to $\left\{x_{e}^{*}, f_{i, e}^{*}\right\}$ and project the $\operatorname{LP}(2)$ onto variables $\left\{z_{I}\right\}$ as follows.

$$
\sum_{e \in E} c_{e} x_{e}^{*}+\min \left\{\sum_{I \subseteq K} \pi(I) z_{I} \mid \sum_{I \subseteq K} z_{I}=1, \gamma_{i} \leq \sum_{I: i \in I} z_{I} \leq \gamma_{i}^{\prime} \forall i \in K, z_{I} \geq 0 \forall I \subseteq K\right\} .
$$

Since $\left\{x_{e}^{*}, f_{i, e}^{*}, z_{i}^{*}\right\}$ is a basic feasible solution to (2), it cannot be written as a convex combination of two distinct feasible solutions to (2). Thus we get that $\left\{z_{I}^{*}\right\}$ cannot be written as a convex combination of two distinct feasible solutions to (7), and hence it forms a basic feasible solution to (7). Since there are $1+2|K|$ non-trivial constraints in $(7)$, at most $1+2|K|$ variables $z_{I}$ can be non-zero in any basic feasible solution of (7). Thus the lemma follows.

Now we prove that LP (2) can be solved in polynomial time. We introduce new variables $\gamma \in[0,1]^{k}$ and obtain the following program. Here the function P is defined in Lemma 4.

$$
\begin{array}{ccl}
\text { Minimize } & \sum_{e \in E} c_{e} x_{e}+\mathrm{P}(\gamma) & \\
\text { Subject to } & \sum_{e \in \delta(T)} f_{i, e} \geq\left(1-\gamma_{i}\right) r_{i}(T) & \forall i \quad \forall T \odot(i, S) \\
& f_{i, e} \leq 1-\gamma_{i} & \forall i \forall e \\
x_{e} \geq f_{i, e} & \forall i \forall e \\
& x_{e}, f_{i, e}, \gamma_{i} \in[0,1] & \forall i \forall e
\end{array}
$$

It is clear that solving (8) is enough to solve (2). Now note that this is a convex program since $\mathrm{P}$ is a convex function. To solve (8), we convert its objective function into a constraint $\sum_{e \in E} c_{e} x_{e}+\mathrm{P}(\gamma) \leq$ OPT where OPT is the target objective value and thus reduce it to a feasibility problem. Now to find a feasible solution using an ellipsoid algorithm, we need to show a polynomial time separation oracle. The separation oracle for the first set of constraints can be reduced to a minimum $u-v$ cut problem using standard techniques. The separation oracle for the remaining constraints is trivial.

The separation oracle for the objective function is given as follows. Given a point $(x, \gamma)=\left\{x_{e}, \gamma_{i}\right\}$ that satisfies $\sum_{e \in E} c_{e} x_{e}+\mathrm{P}(\gamma)>\mathrm{OPT}$, we compute a sub-gradient of the function $\sum_{e \in E} c_{e} x_{e}+\mathrm{P}(\gamma)$ w.r.t. variables $\left\{x_{e}, \gamma_{i}\right\}$. The sub-gradient of $\sum_{e \in E} c_{e} x_{e}$ w.r.t. $x$ is simply the cost vector $c$. The sub-gradient of $\mathrm{P}(\gamma)$ w.r.t. $\gamma$ is computed using Lemma 5 , denote it by $y \in \Re_{+}^{k}$. From the definition of sub-gradient, we have that the sub-gradient $(c, y)$ to the objective function at point $(x, \gamma)$ satisfies

$$
\left(\sum_{e \in E} c_{e} x_{e}^{\prime}+\mathrm{P}\left(\gamma^{\prime}\right)\right)-\left(\sum_{e \in E} c_{e} x_{e}+\mathrm{P}(\gamma)\right) \geq(c, y) \cdot\left(\left(x^{\prime}, \gamma^{\prime}\right)-(x, \gamma)\right) .
$$


Now fix any feasible solution $\left(x^{*}, \gamma^{*}\right)$, i.e., the one that satisfies $\sum_{e \in E} c_{e} x_{e}^{*}+\mathrm{P}\left(\gamma^{*}\right) \leq$ OPT. Substituting $\left(x^{\prime}, \gamma^{\prime}\right)=\left(x^{*}, \gamma^{*}\right)$ in the above equation we get,

$$
\begin{aligned}
0=\mathrm{OPT}-\mathrm{OPT} & >\left(\sum_{e \in E} c_{e} x_{e}^{*}+\mathrm{P}\left(\gamma^{*}\right)\right)-\left(\sum_{e \in E} c_{e} x_{e}+\mathrm{P}(\gamma)\right) \\
& \geq(c, y) \cdot\left(x^{*}, \gamma^{*}\right)-(c, y) \cdot(x, \gamma) .
\end{aligned}
$$

Thus $(c, y)$ defines a separating hyperplane between the point $(x, \gamma)$ and any point $\left(x^{*}, \gamma^{*}\right)$ that satisfies $\sum_{e \in E} c_{e} x_{e}^{*}+\mathrm{P}\left(\gamma^{*}\right) \leq \mathrm{OPT}$. Hence we have a polynomial time separation oracle for the objective function as well.

Thus we can solve (8) using the ellipsoid algorithm. The proof of Lemma 2 is hence complete.

\section{References}

[ABHK09] Aaron Archer, MohammadHossein Bateni, MohammadTaghi Hajiaghayi, and Howard Karloff. A technique for improving approximation algorithms for prize-collecting problems. In Proceedings of the 50th Annual IEEE Symposium on Foundations of Computer Science (FOCS), 2009. To appear.

[AKR95] Ajit Agrawal, Philip Klein, and R. Ravi. When trees collide: an approximation algorithm for the generalized Steiner problem on networks. SIAM J. Comput., 24(3):440-456, 1995.

[Bal89] Egon Balas. The prize collecting traveling salesman problem. Networks, 19(6):621-636, 1989.

[BGSLW93] Daniel Bienstock, Michel X. Goemans, David Simchi-Levi, and David Williamson. A note on the prize collecting traveling salesman problem. Math. Programming, 59(3, Ser. A):413-420, 1993.

[BP89] M. Bern and P. Plassmann. The Steiner problem with edge lengths 1 and 2. Information Processing Letters, 32:171-176, 1989.

[CK09] J. Chuzhoy and S. Khanna. An $O\left(k^{3} \log n\right)$-approximation algorithms for vertex-connectivity network design. In Proceedings of the 50th Annual IEEE Symposium on Foundations of Computer Science (FOCS), 2009. To appear.

[CRW01] Fabian A. Chudak, Tim Roughgarden, and David P. Williamson. Approximate $k$-MSTs and $k$-Steiner trees via the primal-dual method and lagrangean relaxation. In Proceedings of the 8th International Conference on Integer Programming and Combinatorial Optimization (IPCO'01), pages 60-70, London, UK, 2001. Springer-Verlag.

[CVV06] J. Cheriyan, S. Vempala, and A. Vetta. Network design via iterative rounding of setpair relaxations. Combinatorica, 26(3):255-275, 2006.

[FJW01] L. K. Fleischer, K. Jain, and D. P. Williamson. An iterative rounding 2-approximation algorithm for the element connectivity problem. In Proceedings of the 42nd IEEE symposium on Foundations of Computer Science (FOCS'01), pages 339-347, 2001.

$\left[\mathrm{GKL}^{+} 07\right]$ A. Gupta, J. Könemann, S. Leonardi, R. Ravi, and G. Schäfer. An efficient cost-sharing mechanism for the prize-collecting steiner forest problem. In Proceedings of the eighteenth annual ACM-SIAM symposium on Discrete algorithms (SODA '07), pages 1153-1162, Philadelphia, PA, USA, 2007. Society for Industrial and Applied Mathematics.

[GW95] Michel X. Goemans and David P. Williamson. A general approximation technique for constrained forest problems. SIAM J. Comput., 24(2):296-317, 1995.

[Haj08] MohammadTaghi Hajiahayi. Prize-collecting steiner networks via iterative rounding. Submitted., 2008.

[HJ06] Mohammad Taghi Hajiaghayi and Kamal Jain. The prize-collecting generalized Steiner tree problem via a new approach of primal-dual schema. In Proceedings of the Seventeenth Annual ACM-SIAM Symposium on Discrete Algorithms, pages 631-640, New York, 2006. ACM.

[Jai01] K. Jain. A factor 2 approximation algorithm for the generalized Steiner network problem. Combinatorica, 21(1):39-60, 2001. 
[JMP00] David S. Johnson, Maria Minkoff, and Steven Phillips. The prize collecting Steiner tree problem: theory and practice. In Proceedings of the Eleventh Annual ACM-SIAM Symposium on Discrete Algorithms (San Francisco, CA, 2000), pages 760-769, New York, 2000. ACM.

[JV01] Kamal Jain and Vijay V. Vazirani. Approximation algorithms for metric facility location and $k$-median problems using the primal-dual schema and Lagrangian relaxation. J. ACM, 48(2):274-296, 2001.

[KN07] G. Kortsarz and Z. Nutov. Approximating minimum cost connectivity problems, Ch. 58 in Approximation Algorithms and Metahueristics, T. F. Gonzales ed.,. PWS, 2007.

[NSW08] Chandrashekhar Nagarajan, Yogeshwer Sharma, and David P. Williamson. Approximation algorithms for prize-collecting network design problems with general connectivity requirements. In 6th International Workshop on Approximation and Online Algorithms (WAOA'08), pages 174-187, 2008.

[Nut09] Z. Nutov. Approximating minimum cost connectivity problems via uncrossable bifamilies and spidercover decompositions. In Proceedings of the 50th Annual IEEE Symposium on Foundations of Computer Science (FOCS), 2009. To appear.

[RZ05] Gabriel Robins and Alexander Zelikovsky. Tighter bounds for graph Steiner tree approximation. SIAM Journal on Discrete Mathematics, 19(1):122-134, 2005.

[SCRS00] F. S. Salman, J. Cheriyan, R. Ravi, and S. Subramanian. Approximating the single-sink link-installation problem in network design. SIAM J. on Optimization, 11(3):595-610, 2000.

[SSW07] Yogeshwer Sharma, Chaitanya Swamy, and David P. Williamson. Approximation algorithms for prize collecting forest problems with submodular penalty functions. In Proceedings of the eighteenth annual ACM-SIAM symposium on Discrete algorithms (SODA '07), pages 1275-1284, Philadelphia, PA, USA, 2007. Society for Industrial and Applied Mathematics. 